\title{
Qualitätssicherung und Patientensicherheit
}

Die Begriffe «Qualitätssicherung in der Medizin» und «Patientensicherheit» sind in den vergangenen Wochen einmal mehr schlagartig in den Fokus des öffentlichen Interesses gerückt. Dies zu Recht: Eine Gesellschaft, die einen relevanten Anteil der ihr zur Verfügung stehenden Mittel zur solidarischen Gesundheitsversorgung verwendet, hat einen Anspruch darauf, Mängel und Fehler dieses Systems zu kennen. Sie darf erwarten, dass die eingekauften Leistungen die bestmögliche Qualität haben und dass es eine Instanz gibt, die hierüber wacht. Und diese Gesellschaft erwartet dies auch, wie letzthin beispielsweise die Sendung «Arena» des Schweizer Fernsehens gezeigt hat. Ihr Titel: «Wer kontrolliert die Götter in Weiss?»

In der Einsicht, dass Selbstregulation der sinnvollste Weg zur Qualitätssicherung ist, unternimmt die FMH seit Jahren vielfältige Anstrengungen auf diesem Gebiet: Die ganze Weiter- und Fortbildungsordnung, Facharztexamina, die wöchentliche Publikation einer Fortbildungszeitschrift, die Standesordnung mit ihren Gremien - dies alles sind Initiativen im Dienste der Qualität der medizinischen Versorgung. ... Dennoch steht die Medizin erneut am Pranger, suggerieren subalterne Bundesbeamte Horrorzahlen von Behandlungsfehlern und ruft die aufgeschreckte Öffentlichkeit verständlicherweise nach Massnahmen, Handlungen und Kontrollen.

«Qualitätssicherung» und «Patientensicherheit»: Der innere Zusammenhang zwischen den beiden Begriffen scheint auf der Hand zu liegen. Je höher die Qualität der Medizin und der medizinischen Leistungserbringer, desto grösser die Sicherheit für die Patienten. Das stimmt. Doch Qualität in der Medizin ist keine fixe, sondern eine veränderbare Grösse; eine Grösse, die zwischen Anspruchgruppen im Diskurs ausgehandelt wird, Teil eines lernenden Systems und einer
Zusammenarbeit von mehreren Partnern. Qualität in der Medizin ist sehr wohl nachweisbar und beschreibbar, doch lässt sie sich nicht auf Standards und Checklisten reduzieren. * Dies unterscheidet unsere Qualitätssicherungsprozesse auch von solchen der Industrie. Während bei der Herstellung von Schrauben oder Zahnpastatuben die Reduktion der Ausschussware durch Optimierung der Prozessabläufe im Vordergrund steht, darf es im medizinischen Behandlungsprozess keinen «Ausschuss» geben. Natürlich müssen wir alles daran setzen, unsere Prozesse zu optimieren und Fehlerquellen zu vermeiden jeder Fehler ist einer zuviel -, doch der Anspruch auf eine Medizin ohne Fehler bleibt unerreichbare Illusion. Leider ist es so: Tragische Verwechslungen und menschliche Fehlleistungen werden sich auch mit noch soviel Aufwand und Kontrolle nie ganz vermeiden lassen.

Für die FMH bedeutet dies keineswegs, dass damit wieder zum «courrant normal» überzugehen sei. Wir werden unsere Anstrengungen im Bereich von Versorgungs- und Outcome-Analysen weiter intensivieren, zu Unterstützungs- und Verbesserungsmassnahmen Hand bieten, wo wir können, und hierzu auch die nötige Verbindlichkeit einfordern. Wir sind aber auch aufgefordert, das Augenmass zu behalten: Der berechtigte Anspruch der Patientensicherheit darf weder in bürokratischem Leerlauf enden, noch darf er Innovation und wissenschaftlichen Fortschritt der klinischen Medizin gefährden oder verhindern. Denn wenn die klinische Medizin in unserem Land zunehmend nur noch im wissenschaftlichen Windschatten anderer Zentren segeln könnte, ginge wiederum auch für die Qualität sehr viel verloren.

Ludwig T. Heuss, Mitglied Zentralvorstand FMH, Ressortverantwortlicher Daten, Demographie, Qualität DDQ aller Kräfte im ärztlichen Umfeld. Schweiz Ärztezeitung 2005;86(18) 1055-9. 


\section{Assurance-qualité et sécurité des patients}

Les termes «assurance-qualité en médecine» et «sécurité des patients» se sont une fois de plus subitement retrouvés dans le collimateur de l'opinion publique au cours de ces dernières semaines. A juste titre d'ailleurs. En effet, une société qui emploie une part importante des moyens financiers dont elle dispose pour promouvoir des soins de santé solidaires a le droit de connaître les erreurs et les lacunes de ce système. Elle est également en droit d'attendre que les prestations qu'elle acquiert soient de la meilleure qualité possible et qu'une instance veille au respect de ce principe. Et cette attente est bien présente, comme le montre la récente diffusion sur la chaîne de télévision suisse alémanique de l'émission «Arena» dont le titre «Qui contrôle les dieux en blouse blanche?» souligne ce propos.

Convaincue que l'autorégulation est la méthode d'assurance-qualité la plus judicieuse, la FMH poursuit depuis plusieurs années ses nombreux efforts en ce sens. La réglementation de la formation postgraduée et continue dans son ensemble, les examens de spécialiste, la publication hebdomadaire d'un journal de formation continue, le Code de déontologie et ses divers organes, sont autant d'initiatives visant à garantir la qualité des soins médicaux. Mais malgré cela, la médecine se retrouve une nouvelle fois clouée au pilori par des fonctionnaires fédéraux subalternes qui citent des chiffres catastrophiques d'erreurs médicales et par une opinion publique inquiète qui réclame, on la comprend, des mesures, des actes, des contrôles.

«Assurance-qualité» et «sécurité des patients», la relation intrinsèque entre ces deux notions semble évidente. Il va de soi que plus la qualité de la médecine et des fournisseurs de prestations va croissant, plus la sécurité des patients est élevée. Mais la qualité médicale n'est pas une grandeur fixe, elle varie. En effet, elle est négociée entre les différents groupes d'intérêts, fait partie intégrante d'un système en formation et résulte de la collaboration entre différents partenaires. La qualité médicale peut aisément être prouvée et décrite, mais elle ne saurait être réduite à une série de standards ou de listes de contrôle*. Voilà ce qui différencie nos processus d'assurancequalité de ceux de l'industrie. Alors que dans la production de vis ou de tubes de dentifrice, il est possible de réduire le nombre de produits défectueux par une optimalisation des processus, les procédés thérapeutiques ne sauraient eux tolérer aucune erreur. Nous devons bien entendu tout faire pour optimiser nos processus et éviter toute source d'erreur, car chaque faute médicale est une faute de trop. L'exigence d'une médecine sans aucune erreur reste néanmoins illusoire. Il faut malheureusement admettre que les confusions tragiques ou les erreurs humaines ne pourront jamais être entièrement évitées, et ce malgré tous les efforts fournis et toutes les procédures de contrôle instaurées.

Pour la FMH, cela ne signifie en aucun cas qu'il faille faire comme si rien ne s'était passé. Nous continuerons à intensifier nos activités d'analyse des soins et des résultats, à soutenir plus que jamais la prise de mesures de soutien et d'amélioration partout où cela semble possible, et à prendre et exiger les engagements nécessaires. Nous sommes cependant également tenus à la mesure, car l'exigence, si légitime soit-elle, de sécurité des patients ne saurait devenir une impasse bureaucratique ni mettre en péril ou entraver les innovations et les progrès de la médecine clinique. En effet, si la médecine clinique ne devait, dans notre pays, pouvoir évoluer que dans l'ombre d'autres domaines scientifiques, ce serait bien, pour finir, la qualité qui risquerait d'y perdre.

Ludwig T. Heuss, Membre du Comité central de la FMH, Responsable du domaine Données, démographie et qualité (DDQ)

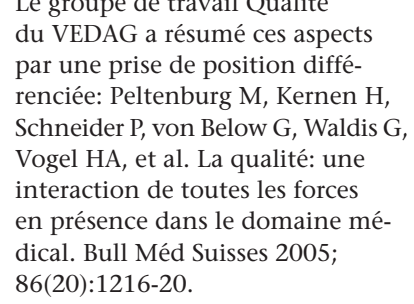

en présence dans le domaine médical. Bull Méd Suisses 2005 86(20):1216-20. 